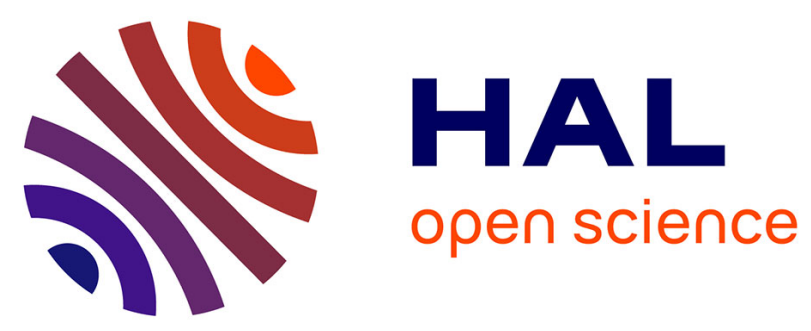

\title{
Decision for adjuvant treatment in stage II colon cancer based on circulating tumor DNA:The CIRCULATE-PRODIGE 70 trial
}

Julien Taïeb, Léonor Benhaim, Pierre Laurent Puig, Karine Le Malicot, Jean François Emile, Flore Geillon, David Tougeron, Sylvain Manfredi, Marion Chauvenet, Valérie Taly, et al.

\section{To cite this version:}

Julien Taïeb, Léonor Benhaim, Pierre Laurent Puig, Karine Le Malicot, Jean François Emile, et al.. Decision for adjuvant treatment in stage II colon cancer based on circulating tumor DNA:The CIRCULATE-PRODIGE 70 trial. Digestive and Liver Disease, 2020, 52 (7), pp.730-733. 10.1016/j.dld.2020.04.010 . inserm-02992943

\section{HAL Id: inserm-02992943 https://www.hal.inserm.fr/inserm-02992943}

Submitted on 10 Nov 2020

HAL is a multi-disciplinary open access archive for the deposit and dissemination of scientific research documents, whether they are published or not. The documents may come from teaching and research institutions in France or abroad, or from public or private research centers.
L'archive ouverte pluridisciplinaire $\mathbf{H A L}$, est destinée au dépôt et à la diffusion de documents scientifiques de niveau recherche, publiés ou non, émanant des établissements d'enseignement et de recherche français ou étrangers, des laboratoires publics ou privés. 
"Decision for adjuvant treatment in stage II colon cancer based on circulating tumor DNA:The CIRCULATE-PRODIGE 70 trial"

Julien Taïeb MD-PhD 1 , Léonor Benhaim MD-PhD², Pierre Laurent Puig MD-PhD Karine Le Malicot Master24, Jean François Emile MD-PhD ${ }^{5}$, Flore Geillon PhD David Tougeron ${ }^{6} \mathrm{MD}-\mathrm{PhD}$, Sylvain Manfredi MD-PhD ${ }^{4,8}$, Marion Chauvenet MD-PhD ${ }^{7}$, Valerie Taly ${ }^{8} \mathrm{PhD}$, Côme Lepage MD-PhD ${ }^{4,9 *} \&$ Thierry André MD-PhD ${ }^{10 *}$.

\section{*equally contributed}

1. Department of gastroenterology and GI Oncology, Georges-Pompidou European Hospital, AP-HP; Sorbonne Paris cité, Université de Paris, Paris, France

2. Département de chirurgie viscérale oncologique, Gustave Roussy, Villejuif, France

3. Pole biologie, Hospital European George Pompidou, Paris, Île-de-France, France

4. Fédération Francophone de Cancérologie Digestive; EPICAD INSERM LNC-UMR 1231, University of Burgundy and Franche Comté, Dijon, France

5. EA4340, Ambroise Pare Hospital, Beuvry, Hauts-de-France, France

6. Gastroenterology Department and Medical Oncology Department, Poitiers University Hospital, Poitiers, France

7. Department of Gastroenterology, Groupement hospitalier Sud, Hospices civils de Lyon, Pierre Bénite, France

8. Centre de Recherche des Cordeliers, INSERM, CNRS, Sorbonne Université, USPC, Université de Paris, Equipe labellisée Ligue Nationale contre le cancer, Paris, France.

9. Department of digestive oncology University hospital Dijon; University of Burgundy and Franche Comté, Dijon, France

10. Sorbonne Université and Hôpital Saint Antoine, Paris, France

Corresponding author:

Pr Julien Taieb

Adress: HEGP, 20 rue Leblanc, 75015 Paris, France

Email: jtaieb75@gmail.com

Phone number: 0033156093551

Conflict of interest: JT has received honoraria for speaker or advisory role from Sanofi, Roche, Merck, Amgen, Sirtex, Servier, Lilly, Celgene and MSD. 
PLP is a consultant/advisory board member for Merck Serono, Amgen, Boehringer Ingelheim, Biocartis, Roche, Bristol-Myers Squibb and MSD.

$\mathrm{CL}$ has served in a consulting/advisory role and or received honoraria for, Amgen, Pierre Fabre, Novartis, AAA.

TA has served in a consulting/advisory role and or received honoraria for, Amgen, Bristol-Myers Squibb, Chugai, Clovis, Halliodx, MSD Oncology, Pierre Fabre, Roche/Ventana, Sanofi, Servier and has received travel, accommodations, and expenses from Roche/Ventana, MSD Oncology, and Bristol-Myers Squibb.

LB, KLM, JFE, FG, DT, SM, MC have nothing to disclose.

\section{Authors contributed equally to this manuscript.}

This work was partially supported by the A.R.C.A.D (Aide et Recherche en Cancérologie Digestive) foundation and a national grant from the French national cancer institute (INCa, PHRC 2019).

Keywords: Colon cancer; adjuvant; circulating tumor DNA; methylated biomarker

Word: 1772 


\section{Abstract \\ Background}

Adjuvant treatment for stage II colon cancer remains debated. Finding a tool to select patients at risk for disease recurrence may help the clinical decision. Circulating tumor DNA (ctDNA) has been reported recently as a potential predictive marker for disease recurrence. We thus aim to test its ability to better select stage II colon cancer patients for adjuvant therapy.

\section{Methods}

This national, phase III trial (NCT 2019-000935-15) conducted in more than 100 centers in France, plans to screen around 2640 patients in order to randomize (2:1; minimization method) 198 ctDNA positive patients. Patients aged 18 to 75 years with ECOG performance status $\leq 1$ with $\mathrm{R} 0$ surgical resection of a pT3-T4aN0 colon or high rectum adenocarcinoma will be randomized within 63 days after curative-intent surgery, to adjuvant mFOLFOX6 (oxaliplatin $85 \mathrm{mg} / \mathrm{m}^{2}$, leucovorin $400 \mathrm{mg} / \mathrm{m}^{2}$, and 5FU bolus $400 \mathrm{mg} / \mathrm{m} 2$ then 5FU Continuous infusion $2.4 \mathrm{~g} / \mathrm{m}^{2}$ ) every two weeks for 12 cycles or observation. Patients will be followed for maximum 7 years. A gain of $17.5 \%$ in 3-yr disease free survival (DFS) is expected $(42.5 \%$ in the experimental arm vs. $25 \%$ in the control arm; HR:0.62; $\alpha, 5 \%$ [two-sided log-rank test]; $1-\beta, 80 \%$ ). Secondary endpoints include 2-yr DFS, overall survival, and toxicity.

The study was opened to inclusion in the end of January 2020. 


\section{Rational}

With an estimated number of 446,000 new patients per year in Europe and 44,000 new patients registered each year in France, colorectal cancer (CRC) is the second most common cancer in Europe and the third in France. It leads to death around 214,000 patients per year in Europe (2012) - accounting for $13 \%$ of all cancer deaths [1].

Patients with colon cancer (CC) represent approximately $68 \%$ of all CRC patients including $36 \%$ of stage II [2]. Death after recurrence following stage II colon cancer contributes approximately to $16 \%$ of the mortality from colon cancer [3]. This represents about 1,600 deaths per year in France- similar to all deaths from melanoma- and 23,000 deaths per year in Europe.

In stage II colon cancer, adjuvant therapy with 5-Fluorouracil and leucovorin- if administered to all patients - results in merely 3\% improvement in 5-year survival [4]. Although various histo-prognostic factors may influence the prognosis, the only widely accepted method to drive the treatment decision is the screening for MSI status which predicts a good prognosis and potentially no benefit from adjuvant chemotherapy [5].

A Cochrane review has demonstrated that although associated with a small benefit in disease free survival (hazard ratio $0.83[95 \% \mathrm{Cl} 0.75-0.91]$, adjuvant systemic chemotherapy does not result into a significant improvement in overall survival (hazard ratio 0.96 [0.87-1.05]). The authors confirmed the need to define which risk features should be used to select patients with stage II colon cancer for adjuvant therapy [6]. A fluoropyrimidine based adjuvant therapy is therefore not the standard of care in stage II colon cancer according to the TNCD (French guidelines, http://www.tncd.org) nor ESMO guidelines but can be considered in patients with a higher risk of disease recurrence defined by clinical and pathological factors. In proficient MMR (pMMR) tumors, adjuvant chemotherapy is currently discussed individually for patients with T4-stage, bowel perforation, number of nodes examined $<10$ or 12 , and less consuensually for patients with vascular, perineural or lymphatic invasion, undifferentiated tumor and bowel obstruction. Six months of oxaliplatinbased treatment is an option as adjuvant chemotherapy for patients with high risk stage II CC especially T4, inadequate nodal harvest and perforation [2]. Overall, the 
T4bN0 tumor stage (tumor directly invades or is adherent to other organs or structures) is one of the strongest prognostic factor, associated with reported survivals of $58.4 \%$ (5-year relative survival) and $45.7 \%$ (5 year overall survival) [7-8]. Furthermore, beyond prognostic, the value of these factors in predicting the benefit of adjuvant chemotherapy is not proven.

With the increased sensitivity of current next generation sequencing (NGS) techniques, ctDNA can be detected by sequencing tumor specific mutations as i.e. TP53, APC, KRAS or NRAS that are present in the vast majority of patients with colorectal cancer. It can also be detected with simpler techniques such as the detection of 2 methylated markers WIF1 and NPY by digital droplet PCR after bisulfitation of the DNA extracted from plasma accordingly to a method developed and validated in colorectal cancer patients [9-12]. Briefly, it has been demonstrated that these markers are present in most tumor samples independently of the disease stage and that the monitoring of methylated ctDNA (Met-ctDNA) using these markers is as efficient as when using patient tumor mutation-specific assays $[10,11]$ and that it could also be used as a control of presence of ctDNA in advanced cancer [12]. Moreover, Met-ctDNA concentration, measured by these markers, was shown to be prognostic of disease progression and patient survival, both in advanced and localized disease. Finally, early decrease of ctDNA concentration bas been demonstrated to be predictive of response to chemotherapy in CC patients in the metastatic setting [11].

In a recent observational study in stage II colon cancer, the detection of residual circulating tumor DNA (ctDNA) after complete surgical resection was highly predictive for tumor recurrence. The estimated recurrence free survival in ctDNA positive patients not treated with chemotherapy was $22 \%$ compared to $90 \%$ in ctDNA negative [13]. Comparable results have been observed for stage III colon cancer patients [14]. More recently, our group has shown that ctDNA was also highly predictive of recurrence in stage III patients coming from a large phase III prospective trial. We also observed that 3 months of adjuvant FOLFOX seemed insufficient in ctDNA+ patients, compared to 6 months treatment underlining the potential guidance that ctDNA might bring to our therapeutic strategy in the adjuvant setting [15] .

The current CIRCULATE-PRODIGE 70 trial aims to evaluate the efficacy of risk stratification in stage II patient based on the post-operative ctDNA detection and the 
efficacy of adjuvant therapy in patients with positive detection of ctDNA. The patients with negative ctDNA detection will be randomized to be followed within the trial or not, as following all negative patients within the trial will not be possible with the budget allocated. The generated knowledge on this patients population may enable to tailor the surveillance programs accordingly.

\section{Patients and study design}

The CIRCULATE-PRODIGE 70 study is an open-label randomized (2:1) phase III trial conducted in France, sponsored by Dijon Bourgogne University Hospital and coordinated operationally by the FFCD data center, comparing adjuvant mFOLFOX6 (oxaliplatin $85 \mathrm{mg} / \mathrm{m}^{2}$, leucovorin $400 \mathrm{mg} / \mathrm{m}^{2}$, and 5 -FU bolus $400 \mathrm{mg} / \mathrm{m}^{2}$ then 2.4 $\mathrm{g} / \mathrm{m}^{2}$ continuous infusion over $46 \mathrm{~h}$ ) every two weeks for 24 weeks (12 cycles) to observation.

Eligible patients, $\geq 18$ years and $<76$ years, ECOG $\leq 1$, curatively $R 0$ resected within 63 days before randomization will have to be positive for ctDNA detection 2 weeks or more after the surgical procedure and before week 8 to allow the randomization in due course.

The ctDNA status will be assessed by digital droplet PCR after bisulfitation of the DNA extracted from the plasma, by the detection of 2 methylated markers (WIF1 and NPY) according to a method developed and validated for colorectal cancer [9-12]. Eligibility criteria are shown in table 1.

TABLE 1: main eligibility criteria

\section{Main inclusion criteria}

- Colon and high rectum cancer stage II excluding low and medium rectal cancers (tumor location $\geq 12 \mathrm{~cm}$ from the anal verge by endoscopy and/or above the peritoneal reflection at surgery),

- without gross or microscopic evidence of residual disease after surgery with curative intent Randomization planned up to 8 weeks after curative resection

- Age $\geq 18$ years and $\leq 75$ years

- ECOG performance Status (ECOG-PS) <2

- Signed written informed consent obtained prior to any study specific procedures

- Available ctDNA analysis

- Available preoperative CT-scan within 21 days before surgery 


\section{Main non-inclusion criteria}

- Known DPD deficiency

- T4b tumors

- Radio or radiochemotherapy for rectal tumors

- Contraindication to chemotherapy (inadequate bone marrow, hepatic, renal functions)

- Comorbidity influencing the prognosis of the patients (i.e. active secondary cancer or others)

- Participation to another interventional study for postoperative therapy

The randomization is adjusted for the following stratification factors: emergency surgery (yes vs no); T4a stage (yes vs no) and study site.

Eligible ctDNA positive patients will be randomized using a 2:1 ratio ("Chemotherapy" or "Follow-up within the trial"). Eligible ctDNA negative patients will be randomized using a 1:4 ratio ("Follow-up within the trial" or "Follow-up outside the trial"). In total 2640 patients will be screened for inclusion and exclusion criteria. Assuming $25 \%$ of patients not filling eligibility criteria, around 2640 patients should be screened for ctDNA assessment, 554 will be followed up within the study and $198 \mathrm{ctDNA}+$ will be randomized to receive or not adjuvant chemotherapy (Figure 1).

This study (clinicaltrials.gov NCT 2019-000935-15) is performed in accordance with the Declaration of Helsinki and Good Clinical Practice Guidelines. A French ethics committee approved the study. All patients have to provide written informed consent before starting the study.

\section{Endpoints and Assessments}

The primary objective is DFS rate at 3 years defined as the time from the date of randomization up to the date of first local, regional or distant relapse, second CRC, or death from any cause including treatment-related death. Secondary objectives include DFS at 2 years, time to recurrence (TTR), Overall Survival (OS), and toxicities according to the $\mathrm{NCI}-\mathrm{CTCAE}$ version 4.03 comparisons between patients assigned to FOLFOX or to observation.

Tumor assessment is recommended to be performed before surgery and every 6 months, with thoraco-abdominal and pelvic CT-scan after surgery for 5 years. The CEA level will be assessed according to the same schedule. After 5 years postsurgery, a thoraco-abdominal and pelvic CT-scan is recommended every year. 
DFS and OS in ctDNA positive versus ctDNA negative patients, without adjuvant treatment will also be compared.

Finally, the added value of ctDNA on classical prognostic factors for stage II colon cancer will be tested together with the predictive value of clinical/pathological and biological factors for adjuvant treatment efficacy in ctDNA+ stage II colon cancer.

\section{Statistical analysis plan}

The study will include patients during 48 months and the follow-up will continue until 36 months after the last randomized patient (for evaluation of DFS at 3 years). Two interim efficacy analyses for futility and efficacy purposes are planned after $33 \%$ of events and $60 \%$ of events. To reach an overall alpha of $5 \%$, the alpha spend at first stage is 0.00019 and 0.00762 at second stage. We expect a $17.5 \%$ gain for DFS at 3-year in the experimental arm (FOLFOX) compared with the reference arm (observation), $42.5 \%$ versus $25 \%$, respectively; HR:0.617). In order to significantly conclude with $80 \%$ power and $5 \%$ alpha level in a two-sided log-rank test, we need to obtain at least 154 events and 198 patients have to be analyzed.

\section{Ancillary study: biomarker analysis}

All patients enrolled in this trial will be proposed to consent for their participation to a biological ancillary study. Prognostic and predictive biomarkers can be studied both on blood and on tumor-derived samples.

In addition to ctDNA status, this study will allow us to better assess in a large series of patients the prognostication of stage II colon cancer for which little is known compared to stage III.

We aim to define a nomogram using different prognostic factors focused on the characterization of the different tumor molecular phenotype including (but not limited to): 
- after DNA extraction from formalin fixed paraffin embedded tumor the Mismatch repair deficiency phenotype (dMMR), the $\mathrm{CpG}$ island methylator phenotype (CIMP) and the most frequent mutations that occur in colon cancer.

- The molecular subtype (CMS) by using the nanostring technology after RNA extraction from the same sample tumor

- And finally the tumor microenvironment characterized by immunochemistry technics with a focus on Immunoscore ${ }^{\mathrm{TM}}$ which will be determined according to HalioDX test plus other scores under development taking into account the stromal reaction observed in the tumor (DGmune).

Theses molecular characteristics will be correlated to ctDNA status, time to recurrence, DFS and OS. The univariate and multivariate analyses will be performed in order to determine the best model to predict DFS in this homogenous group of stage II colorectal tumor including or not the ctDNA status.

\section{Conclusion}

CIRCULATE is a phase III randomized trial evaluating, in patients with detectable ctDNA after the curative surgical resection of a stage II colon or high rectum cancer, the benefit of mFOLFOX6 adjuvant therapy compared to surveillance alone. We plan to enroll a total of 554 ctDNA patients. The 198 ctDNA positive patients will be randomized (2:1) between mFOLFOX6 or observation and the remaining 356 ctDNApatients will be followed up to improve our knowledge on the prognosis value of ctDNA in stage II colon cancer patients.

The study was opened to inclusion in the end of January 2020. Overall, 100 centers will be opened in France. The end of inclusions is scheduled for December 2024.

\section{Acknowledgements}

This work was partially supported by the A.R.C.A.D (Aide et Recherche en Cancérologie Digestive) foundation and a national grant from the French national cancer institute (INCa, PHRC 2019). 


\section{References}

[1] Ferlay J, et al. Cancer incidence and mortality patterns in Europe: Estimates for 40 countries in 2012. Eur. J. Cancer. 2013;49:1374-1403

[2] Taieb J, André T, Auclin E.Refining adjuvant therapy for non-metastatic colon cancer, new standards and perspectives. Cancer Treat Rev. 2019 Feb 26;75:111.

[3] Guyot $F$, et al. Time trends in the treatment and survival of recurrences from colorectal cancer. Ann Oncol. 2005;16:756-761

[4] Quasar-Collaborative-Group, et al. Adjuvant chemotherapy versus observation in patients with colorectal cancer: a randomised study. Lancet. 2007;370:20202029

[5] Sargent DJ, et al. Defective mismatch repair as a predictive marker for lack of efficacy of fluorouracil-based adjuvant therapy in colon cancer. J. Clin. Oncol. 2010;28:3219-3226

[6] Figueredo A, et al. Adjuvant therapy for completely resected stage II colon cancer. Cochrane database Syst Rev. 2008;CD005390

[7] Tournigand C, et al. Adjuvant therapy with fluorouracil and oxaliplatin in stage II and elderly patients (between ages 70 and 75 years) with colon cancer: subgroup analyses of the Multicenter International Study of Oxaliplatin, Fluorouracil, and Leucovorin in the Adjuvant Treatment of Colon Cancer trial. J Clin Oncol. 2012; 3353-3360

[8] American Joint Committee on Cancer. AJCC Cancer Staging Manual. 7th edition, 2010. ISBN: 978-0-387-88440-0

[9] Roperch J-P, et al. Aberrant methylation of NPY, PENK, and WIF1 as a promising marker for blood-based diagnosis of colorectal cancer. BMC Cancer. 2013;13:566

[10] Garrigou S, et al. A Study of Hypermethylated Circulating Tumor DNA as a Universal Colorectal Cancer Biomarker. Clin Chem. 2016;62:1129-1139

[11] Garlan F, et al. Early Evaluation of Circulating Tumor DNA as Marker of Therapeutic Efficacy in Metastatic Colorectal Cancer Patients (PLACOL Study). Clin Cancer Res. 2017;23:5416-5425

[12] Bachet JB, et al. RAS mutation analysis in circulating tumor DNA from patients with metastatic colorectal cancer: the AGEO RASANC prospective multicenter study. Ann Oncol. 2018;29:1211-1219 
[13] Tie J, et al. Circulating tumor DNA analysis detects minimal residual disease and predicts recurrence in patients with stage II colon cancer. Sci Transl Med. 2016;8:346ra92

[14] Reinert J, et al. Analysis of Plasma Cell-Free DNA by Ultradeep Sequencing in Patients With Stages I to III Colorectal Cancer. JAMA Oncol. 2019

[15] Taieb J, et al. Analysis of circulating tumour DNA (ctDNA) from patients enrolled in the IDEA-FRANCE phase III trial: Prognostic and predictive value for adjuvant treatment duration. ANNALS OF ONCOLOGY. 2019. 30, 867-867 